\title{
Thermal evolution of rocky exoplanets with a graphite outer shell
}

\author{
Kaustubh Hakim ${ }^{1,2,3}$, Arie van den Berg ${ }^{3,4}$, Allona Vazan ${ }^{2,5,6}$, Dennis Höning ${ }^{3,7}$, \\ Wim van Westrenen ${ }^{3}$, and Carsten Dominik ${ }^{2}$
}

${ }^{1}$ Center for Space and Habitability, University of Bern, Gesellschaftsstrasse 6, 3012 Bern, Switzerland e-mail: kaustubh.hakim@csh.unibe.ch

2 Anton Pannekoek Institute for Astronomy, University of Amsterdam, Science Park 904, 1098 XH Amsterdam, The Netherlands

${ }^{3}$ Department of Earth Sciences, Vrije Universiteit, De Boelelaan 1085, 1081 HV Amsterdam, The Netherlands

${ }^{4}$ Department of Earth Sciences, Utrecht University, Princetonlaan 8a, 3584 CB Utrecht, The Netherlands

${ }^{5}$ Racah Institute of Physics, The Hebrew University, Jerusalem 91904, Israel

${ }^{6}$ Institute for Computational Science, Center for Theoretical Astrophysics and Cosmology, University of Zürich, 8057 Zürich, Switzerland

${ }^{7}$ Origins Center, University of Groningen, Nijenborgh 7, 9747 AG Groningen, The Netherlands

Received 17 April 2019 / Accepted 10 September 2019

\begin{abstract}
Context. The presence of rocky exoplanets with a large refractory carbon inventory is predicted by chemical evolution models of protoplanetary disks of stars with photospheric $\mathrm{C} / \mathrm{O}>0.65$, and by models studying the radial transport of refractory carbon. Highpressure high-temperature laboratory experiments show that most of the carbon in these exoplanets differentiates into a graphite outer shell.

Aims. Our aim is to evaluate the effects of a graphite outer shell on the thermal evolution of rocky exoplanets containing a metallic core and a silicate mantle.

Methods. We implemented a parameterized model of mantle convection to determine the thermal evolution of rocky exoplanets with graphite layer thicknesses up to $1000 \mathrm{~km}$.

Results. We find that because of the high thermal conductivity of graphite, conduction is the dominant heat transport mechanism in a graphite layer for long-term evolution ( $>200 \mathrm{Myr}$ ). The conductive graphite shell essentially behaves like a stagnant lid with a fixed thickness. Models of Kepler-37b (Mercury-size) and a Mars-sized exoplanet show that a planet with a graphite lid cools faster than a planet with a silicate lid, and a planet without a stagnant lid cools the fastest. A graphite lid needs to be approximately ten times thicker than a corresponding silicate lid to produce similar thermal evolution.
\end{abstract}

Key words. planets and satellites: terrestrial planets - planets and satellites: interiors - planets and satellites: physical evolution planets and satellites: composition - planets and satellites: surfaces - methods: numerical

\section{Introduction}

Rocky exoplanets appear to be ubiquitous around all types of planet-hosting stars in our galaxy (Petigura et al. 2018). Massradius relations of rocky exoplanets hint at a large variety in their composition ranging from rock-iron compositions to ice-water worlds (e.g., Valencia et al. 2006; Seager et al. 2007; Wagner et al. 2011; Hakim et al. 2018a). Other indications about their compositional diversity come from spectroscopic observations of their host stars, which show a range in photospheric elemental ratios, especially $\mathrm{Mg} / \mathrm{Si}$ and $\mathrm{C} / \mathrm{O}$ (e.g., Bond et al. 2008; Delgado Mena et al. 2010). Chemical evolution simulations of refractory materials, which are the building blocks of rocky planets, in protoplanetary disks of these planet-hosting stars widen their compositional diversity even further, in particular in terms of their refractory C/O ratio (e.g., Bond et al. 2010; Carter-Bond et al. 2012; Moriarty et al. 2014; Dorn et al. 2019).

Planet-hosting stars with molar $\mathrm{C} / \mathrm{O}>0.65$ (cf. $\mathrm{C} / \mathrm{O}_{\text {Sun }} \sim$ 0.54) are capable of producing short-period rocky exoplanets abundant in carbon (Moriarty et al. 2014). Although the accuracy of photospheric $\mathrm{C} / \mathrm{O}$ ratio measurements of stars in the solar neighborhood is still under debate (e.g., Delgado Mena et al. 2010; Petigura \& Marcy 2011; Nakajima \& Sorahana 2016; Brewer et al. 2016), there is a large spread in the reported $\mathrm{C} / \mathrm{O}$ ratios ranging from 0.2 to 1.6 . This hints that a substantial fraction of stars still may have photospheric $\mathrm{C} / \mathrm{O}$ ratios exceeding 0.65 and consequently they are likely to host carbon-enriched rocky exoplanets. Even in our solar system, refractory carbon is not rare. Graphite and diamond have been observed in ureilite parent body meteorites (Nabiei et al. 2018). Graphite is also speculated to be present on the surface of Mercury (Peplowski et al. 2016). The chemical-dynamical simulations of Carter-Bond et al. (2012) accounting for giant planet migration show that rocky planets around high $\mathrm{C} / \mathrm{O}$ stars can contain, in addition to iron and silicates, up to $47 \mathrm{wt} \%$ carbon in weight in the form of graphite, diamond, silicon carbide, and titanium carbide. Furthermore, if radial transport of dust containing refractory carbon is efficient, carbon fractions significantly larger than observed in terrestrial planets of the solar system should be possible (Klarmann et al. 2018).

Because pressures in planetary interiors are orders of magnitude higher than pressures in protoplanetary disks, the refractory material formed in protoplanetary disks undergoes highpressure, high-temperature processing, thereby ensuing changes in mineralogy. Laboratory experiments show that carbonenriched rocky exoplanets containing an iron-rich core and a silicate-rich mantle can dissolve carbon only up to an order of a percent by weight and that graphite (and diamond depending 
on the pressure) is the dominant carbon-bearing mineral (Hakim et al. 2019). Silicon carbide is stable only under extremely reducing conditions (Hakim et al. 2018b). Titanium carbide, even if present, is expected in small amounts because of the relatively low elemental abundance of titanium. Hence, we do not consider these carbides in the context of this study. Since graphite is $25-40 \%$ lower in density than regular silicate minerals and silicate melts, graphite is expected to float on a magma ocean and consequently form an outer shell in carbon-enriched planets assuming efficient density-driven segregation (e.g., Keppler \& Golabek 2019).

After planet formation and differentiation, the heat locked up in rocky planetary interiors, which stems from, for example, accretion and differentiation processes, core contraction, latent heat of solidification, and radioactive decay, is gradually released to space. Physical properties control the heat transport by convection, conduction, and radiation; these properties include the planet radius, the interior layer thicknesses, and rock and mineral properties such as thermal conductivity and viscosity. In rocky planets, radiation has a negligible role to play and heat is transported mainly through conduction and convection. The contribution of convective heat transport is expressed by the Nusselt number, which increases with the vigor of thermal convection from a value of unity for purely conductive heat transport (Schubert et al. 2001).

Thermal evolution and interior dynamics in solar and extrasolar planetary bodies have been studied in detail for Earth-like silicate rock compositions (e.g., Schubert et al. 1979; Spohn 1991; Valencia et al. 2007; van den Berg et al. 2010; Höning \& Spohn 2016; Zhao et al. 2019). Only a few studies have focused on the thermal evolution in planetary layers with nonsilicate mineralogies such as ice (e.g., Deschamps \& Sotin 2001; Deschamps $\&$ Lin 2014, for icy satellites and dwarf planets), water and ice (e.g., Noack et al. 2016, for extrasolar waterworlds) and diamond (e.g., Unterborn et al. 2014, for carbon-enriched exoplanets). The outermost shell determines the efficiency of heat transfer from the interior to the surface and subsequently affects the interior dynamics including the tectonic mode, volcanism, deep volatile cycles, and the presence of a magnetic field (e.g., Schubert et al. 2001; Höning et al. 2019). Consequently, these processes have the potential to affect the habitability of the surface of a planet greatly.

The presence of graphite as an outer shell in carbon-enriched rocky exoplanets presents a unique problem and is likely to influence the planetary dynamics and habitability. In addition to its low density compared to silicate and iron-rich materials, graphite has other peculiar properties including an order of magnitude higher thermal conductivity $\left(20-200 \mathrm{~W} \mathrm{~m}^{-1} \mathrm{~K}^{-1}\right.$, Tyler \& Wilson 1953; Boylan 1996; Hofmeister et al. 2014) than silicates (3-6 W m ${ }^{-1} \mathrm{~K}^{-1}$, Kobayashi 1974; Hofmeister 1999), a high melting temperature of about $4500 \mathrm{~K}$ at all pressures of its stability (Kerley \& Chhabildas 2001; Ghiringhelli et al. 2005), and metal-like specific heat of about $700 \mathrm{~J} \mathrm{~kg}^{-1} \mathrm{~K}^{-1}$ (Boylan 1996). Unterborn et al. (2014) found that the high thermal conductivity of diamond ( $3000 \mathrm{~W} \mathrm{~m}^{-1} \mathrm{~K}^{-1}$, Wei et al. 1993) has a significant impact on planetary cooling; in that study they assumed diamond to be homogeneously mixed with silicates owing to their similar densities. To our knowledge, no study has focused on the thermal evolution of low-mass planets in which carbon differentiates into a graphite shell.

In this paper, our goal is to evaluate, to first order, the effects of a graphite outer shell on the thermal evolution of rocky exoplanets. In Sect. 2, we describe our one-dimensional parameterized thermal evolution model applied to the main layered reservoirs in these planets. In Sect. 3, we first establish the nature of heat transport in the graphite shell. Then we quantify the effects of a conductive lid made of either graphite or silicate on top of the silicate mantle on the thermal evolution of Mars-size and Mercury-size rocky exoplanets. In Sect. 4, we summarize our results and discuss the implications of our results on planets that have lids with non-graphite-like thermal conductivities and planets of different sizes.

\section{Modeling methods}

\subsection{Interior structure}

To model the thermal evolution of a planet with multiple concentric shells, realistic values of input parameters such as the average density of each layer and surface gravity, are required (see Sect. 2.2). These values are determined by computing the planetary interior structure by integrating the equation describing the hydrostatic equilibrium and Poisson's equation from the center to the surface as a function of the radial distance $r$, assuming a spherically symmetric and isotropic dependence of material properties. The equations are written as

$$
\begin{aligned}
& \frac{\mathrm{d} P}{\mathrm{~d} r}=-\rho g, \\
& \frac{\mathrm{d} g}{\mathrm{~d} r}=4 \pi G \rho-2 \frac{g}{r},
\end{aligned}
$$

where $P$ is pressure, $g$ is gravitational acceleration, $G$ is the gravitational constant, and the density $\rho(P)$ is calculated using appropriate equations of state. Since temperature has a small effect on the order of a few percent on density (e.g., Hakim et al. 2018a), we ignore the effect of temperature on material density for interior structure calculations.

For a planet with three concentric shells and a total radius $R_{\text {surf (see Fig. 1), three sets of Eqs. (1) and (2) need to be solved }}$ and require six boundary conditions: $P\left(R_{\text {surf }}\right)=0, g(0)=0$ and four continuity conditions for $P$ and $g$ at the two interfaces of this planet with three layers. Similarly, for a planet with two layers, two sets of Eqs. (1) and (2) are solved with corresponding boundary conditions. Mass is calculated by integrating the mass-continuity equation $\mathrm{d} m / \mathrm{d} r=4 \pi r^{2} \rho$.

To compute material density at a certain pressure, we implemented the equations of state of graphite (Colonna et al. 2011), $\mathrm{MgSiO}_{3}$ (enstatite for $P<25 \mathrm{GPa}$ and $\mathrm{Mg}$-perovskite for $P>$ $25 \mathrm{GPa}$; Stixrude \& Lithgow-Bertelloni 2011), and hcp-Fe (Fei et al. (2016) for $P<234 \mathrm{GPa}$ and Hakim et al. (2018a) for $P>234 \mathrm{GPa}$ ). Comparing the equations of state of graphite, enstatite, and diamond (Dewaele et al. 2008), we verified that graphite is lower in density than enstatite and diamond by $25-40 \%$ at all pressures up to the highest graphite-diamond transition pressure (15 GPa; Ghiringhelli et al. 2005).

Our interior structure calculations for Mars-size and smaller exoplanets show that the material density within a particular layer varies by less than $10 \%$. Hence we assume constant densities for graphite, silicate, and iron layers (Table 1), which are close to our calculated volume-average densities and allow us to analyze model-independent differences in our thermal evolution calculations.

\subsection{Thermal evolution model}

To simulate the thermal evolution of the mantle, we implemented the boundary layer theory analysis of Rayleigh-Bénard convection (Turcotte \& Oxburgh 1967; Stevenson et al. 1983; 


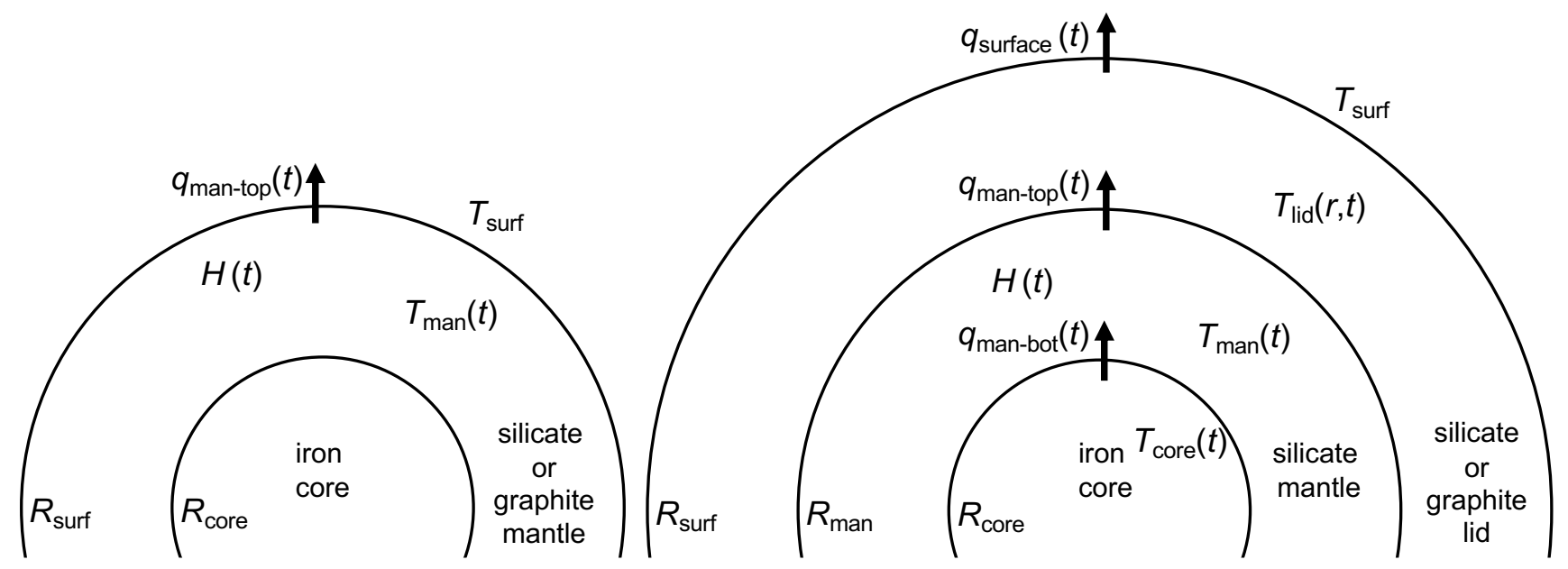

a) Mantle evolution

b) Coupled core-mantle-lid evolution

Fig. 1. (a) Mantle evolution setup (Sect. 2.2.2) for graphite and silicate mantles implemented in Sect. 3.1. (b) Coupled core-mantle-lid evolution setup (Sect. 2.2.3) implemented in Sects. 3.2-3.4.

Schubert et al. 2001). In this section, we first provide equations governing the boundary layer theory and then describe the two types of model setups implemented in this paper.

\subsubsection{Boundary layer theory}

The heat fluxes at the top and bottom of the mantle ( $q_{\text {man-top }}$ and $\left.q_{\text {man-bot }}\right)$ are expressed in terms of the temperature drops across the top and bottom thermal boundary layers $\left(\Delta T_{\text {top }}\right.$ and $\left.\Delta T_{\text {bot }}\right)$, and the Nusselt number $\mathrm{Nu}$ for the entire mantle, i.e.,

$q_{\text {man-j }}(t)=\operatorname{Nu}(t) \frac{k \Delta T_{j}(t)}{h}, \quad j=$ top, bot,

where $k$ is the thermal conductivity of the mantle (either constant or temperature-dependent; Table 1) and $h$ is the height of the mantle. The Nusselt number $\mathrm{Nu}$ is parameterized in terms of the Rayleigh number Ra by a power-law relation (Turcotte \& Schubert 2002),

$\mathrm{Nu}=f_{\mathrm{N}} \mathrm{Ra}^{\beta}$.

Several values between $0.19-0.35$ have been proposed for the power-law exponent $\beta$ depending on geometry, theory, and experiments (Wolstencroft et al. 2009, and references therein). We assumed the classical boundary layer theory exponent $\beta=1 / 3$ from Turcotte \& Oxburgh (1967), which is similar to the $\beta$ for internally heated systems $(0.337 \pm 0.009)$ from Wolstencroft et al. (2009). We took the prefactor value $f_{\mathrm{N}}=0.164$ from Wolstencroft et al. (2009). The Rayleigh number Ra is defined in terms of the mantle properties as

$\operatorname{Ra}(t)=\frac{\alpha g \rho^{2} C_{\mathrm{P}} \Delta T(t) h^{3}}{k \eta(T)}$,

where the super-adiabatic temperature difference $\Delta T$ driving the convection is the sum of the temperature drops across the top and bottom thermal boundary layers $\left(\Delta T=\Delta T_{\text {top }}+\Delta T_{\text {bot }}\right), \alpha$ is the thermal expansivity, $g$ is the gravitational acceleration, $C_{\mathrm{P}}$ is the specific heat capacity, and $\eta(T)$ is temperature-dependent viscosity. The viscosity is given by the Arrhenius law (Schubert et al. 2001),

$\eta(T)=A \exp \left(\frac{E}{R T}\right)$, where $A$ is the rheology prefactor, $E$ is the activation energy, and $R$ is the universal gas constant. For simplicity, we ignore the pressure-dependent $P V$ term, which is additive to the $E$ term in the Arrhenius law ( $V$ is the activation volume and $P$ is the pressure). This is a reasonable approximation in view of other approximations and the limited pressure range considered. The pressure-dependent term $P V$ is small for small planets. For example, for a planet with the radius of $2500 \mathrm{~km}, P V$ is limited to about $10 \%$ of $E$.

\subsubsection{Mantle evolution}

To perform relevant thermal evolution calculations for carbonenriched rocky planets, we implemented two different model setups as shown in Fig. 1. The temperature of the mantle $\left(T_{\operatorname{man}}\right)$ assuming no heat input from the core (Fig. 1a) is given by the conservation of thermal energy (Schubert et al. 2001),

$V_{\mathrm{man}} \rho_{\mathrm{man}} C_{\mathrm{P}, \operatorname{man}} \frac{\mathrm{d} T_{\mathrm{man}}}{\mathrm{d} t}=V_{\mathrm{man}} \rho_{\mathrm{man}} H(t)-A_{\text {man-top }} q_{\text {man-top }}(t)$,

where $H(t)=H_{0} \exp (-t / \tau)$ is the internal heating rate per unit mass due to the radioactive decay with a characteristic exponential decay time $\tau$ (Table 1$), q_{\text {man-top }}(t)$ is the heat flux through the top of the mantle, $A_{\text {man-top }}$ is the surface area of the top of the mantle, $V_{\operatorname{man}}$ is the volume of the mantle, $\rho_{\operatorname{man}}$ is the average mantle density, and $C_{\mathrm{P}, \operatorname{man}}$ is the specific heat capacity of the mantle. The temperature contrast in Eq. (3) for $q_{\text {man-top }}(t)$ is given by $\Delta T_{\text {top }}=T_{\text {man }}-T_{\text {surf }}$, where $T_{\text {surf }}$ is the planet surface temperature.

\subsubsection{Coupled core-mantle-lid evolution}

For models with three layers, core, mantle, and outer shell or lid, we used a coupled core-mantle-lid setup as shown in Fig. 1 b. The thermal evolution of the mantle coupled to that of the core is given by the conservation of thermal energy,

$$
\begin{aligned}
V_{\text {man }} \rho_{\text {man }} C_{\mathrm{P}, \text { man }} \frac{\mathrm{d} T_{\text {man }}}{\mathrm{d} t}= & V_{\text {man }} \rho_{\text {man }} H(t)-A_{\text {man-top }} q_{\text {man-top }}(t) \\
& +A_{\text {man-bot }} q_{\text {man-bot }}(t),
\end{aligned}
$$


Table 1. Input parameters for thermal evolution modeling.

\begin{tabular}{|c|c|c|}
\hline Parameter & Value & Description \\
\hline \multicolumn{3}{|l|}{ Material properties } \\
\hline$\rho_{\text {gra }}\left(\mathrm{kg} \mathrm{m}^{-3}\right)$ & 2300 & Average graphite density (Sect. 2.1) \\
\hline$\rho_{\text {sil }}\left(\mathrm{kg} \mathrm{m}^{-3}\right)$ & 3300 & Average enstatite density (Sect. 2.1) \\
\hline$\rho_{\text {iron }}\left(\mathrm{kg} \mathrm{m}^{-3}\right)$ & 9000 & Average iron density (Sect. 2.1) \\
\hline$C_{\mathrm{P}, \text { gra }}\left(\mathrm{J} \mathrm{K}^{-1} \mathrm{~kg}^{-1}\right)$ & 700 & Specific heat of graphite (Boylan 1996) \\
\hline$C_{\mathrm{P}, \text { sil }}\left(\mathrm{J} \mathrm{K}^{-1} \mathrm{~kg}^{-1}\right)$ & 1250 & Specific heat of silicate (Schubert et al. 2001) \\
\hline$C_{\mathrm{P} \text {,iron }}\left(\mathrm{J} \mathrm{K}^{-1} \mathrm{~kg}^{-1}\right)$ & 550 & Specific heat of iron (Schubert et al. 2001) \\
\hline$\alpha_{\text {gra }}\left(\mathrm{K}^{-1}\right)$ & $3 \times 10^{-5}$ & Thermal expansivity of graphite (Morgan 1972) \\
\hline$\alpha_{\text {sil }}\left(\mathrm{K}^{-1}\right)$ & $3 \times 10^{-5}$ & Thermal expansivity of silicate (Schubert et al. 2001) \\
\hline$k_{\mathrm{gra}}(T)\left(\mathrm{W} \mathrm{m}^{-1} \mathrm{~K}^{-1}\right)$ & $42327 T^{-1.035}+0.00103 T$ & Thermal conductivity of graphite (Hofmeister et al. 2014, Graphite AXM, Table 1) \\
\hline$k_{\text {sil }}\left(\mathrm{W} \mathrm{m}^{-1} \mathrm{~K}^{-1}\right)$ & 5 & Thermal conductivity of silicate (Schubert et al. 2001) \\
\hline$E_{\text {gra }}\left(\mathrm{kJ} \mathrm{mol}^{-1}\right)$ & 209 & Activation energy of graphite (Wagner \& Driesner 1959) \\
\hline$E_{\mathrm{sil}}\left(\mathrm{kJ} \mathrm{mol}^{-1}\right)$ & 300 & Activation energy of silicate (Schubert et al. 2001) \\
\hline$A_{\text {gra,min. }}\left(10^{9} \mathrm{~Pa} \mathrm{~s}\right)$ & 5.3 & Rheology prefactor for graphite (min. shear modulus, Cost et al. 1968) \\
\hline$A_{\text {gra,max. }}\left(10^{9} \mathrm{~Pa} \mathrm{~s}\right)$ & 185 & Rheology prefactor for graphite (max. shear modulus, Min \& Aluru 2011) \\
\hline$A_{\text {sil }}\left(10^{9} \mathrm{~Pa} \mathrm{~s}\right)$ & 160 & Rheology prefactor for silicate (assuming $\eta(1600 \mathrm{~K})=10^{21} \mathrm{~Pa} \mathrm{~s}$ ) \\
\hline \multicolumn{3}{|l|}{ Model properties } \\
\hline$T_{\text {surf }}(\mathrm{K})$ & 700 & Planet surface temperature (Kepler-37b, Barclay et al. 2013) \\
\hline$T_{0, \text { lid-bot }}(\mathrm{K})$ & 1700 & Initial temperature at the bottom of the lid \\
\hline$T_{0, \operatorname{man}}(\mathrm{K})$ & 2000 & Initial mantle temperature \\
\hline$T_{0, \text { core }}(\mathrm{K})$ & 3000 & Initial core temperature \\
\hline$H_{0}\left(10^{-12} \mathrm{~W} \mathrm{~kg}^{-1}\right)$ & 34.5 & Initial internal heating rate of the mantle (Turcotte \& Schubert 2002) \\
\hline$\tau(\mathrm{Gyr})$ & 2.95 & Characteristic decay time of radioactive (Turcotte \& Schubert 2002) \\
\hline
\end{tabular}

Table 2. Planet parameters for thermal evolution modeling.

\begin{tabular}{lcccc}
\hline \hline Model & $R_{\text {surf }}(\mathrm{km})$ & $R_{\text {man }}(\mathrm{km})$ & $R_{\text {core }}(\mathrm{km})$ & $g\left(\mathrm{~m} \mathrm{~s}^{-2}\right)$ \\
\hline Mantle evolution (100 km mantle) & 1600 & - & 1500 & 3.7 \\
Mantle evolution (200 km mantle) & 1700 & - & 1500 & 3.7 \\
Mantle evolution (500 km mantle) & 2000 & - & 1500 & 3.7 \\
Mantle evolution (1000 km mantle) & 2500 & - & 1500 & 3.7 \\
\hline Coupled core-mantle-lid (1 km lid) & 3001 & 3000 & 1500 & 3.5 \\
Coupled core-mantle-lid (50 km lid) & 3050 & 3000 & 1500 & 3.5 \\
Coupled core-mantle-lid (500 km lid) & 3500 & 3000 & 1500 & 3.5 \\
Coupled core-mantle-lid, Kepler-37b (1 km lid) & 2166 & 2165 & 1083 & 2.4 \\
Coupled core-mantle-lid, Kepler-37b (100 km lid) & 2166 & 2066 & 1083 & 2.4 \\
\hline
\end{tabular}

where $H(t)=H_{0} \exp (-t / \tau)$ is the internal heating rate per unit mass due to the radioactive decay with a characteristic exponential decay time $\tau$ (Table 1$), q_{\text {man-top }}(t)$ is the heat flux through the top of the mantle, $A_{\text {man-top }}$ is the area of the top of the mantle, $q_{\text {man-bot }}(t)$ is the heat flux through the bottom of the mantle, $A_{\text {man-bot }}$ is the area of the bottom of the mantle, $V_{\text {man }}$ is the volume of the mantle, $\rho_{\operatorname{man}}$ is the average mantle density, and $C_{\mathrm{P}, \text { man }}$ is the specific heat capacity of the mantle. The temperature contrasts in Eq. (3) for $q_{\text {man-top }}(t)$ and $q_{\text {man-bot }}(t)$ are given by $\Delta T_{\text {top }}=T_{\text {man }}-T_{\text {lid-bot }}$ and $\Delta T_{\text {bot }}=T_{\text {core }}-T_{\text {man }}$, respectively, where $T_{\text {lid-bot }}$ is the temperature at the bottom of the lid.

The core is modeled as a heat reservoir with temperature $T_{\text {core }}$ and its thermal evolution is described by another equation for the conservation of thermal energy,

$V_{\text {core }} \rho_{\text {core }} C_{\mathrm{P}, \text { core }} \frac{\mathrm{d} T_{\text {core }}}{\mathrm{d} t}=-A_{\text {man-bot }} q_{\text {man-bot }}(t)$, where $V_{\text {core }}$ is the volume of the core, $\rho_{\text {core }}$ is the average core density, and $C_{\mathrm{P} \text {,core }}$ is the specific heat capacity of the core.

We modeled the outer shell or lid as a purely conductive static medium. Assuming a spherically symmetric temperature distribution of the lid, the partial differential equation (PDE) for time-dependent conductive heat transport (Schubert et al. 2001) can be written as

$\rho_{\mathrm{lid}} C_{\mathrm{P}, \mathrm{lid}} \frac{\partial T_{\mathrm{lid}}}{\partial t}=\frac{1}{r^{2}} \frac{\partial}{\partial r}\left(r^{2} k_{\mathrm{lid}} \frac{\partial T_{\mathrm{lid}}}{\partial r}\right)$,

where $T_{\text {lid }}(r, t)$ is the lid temperature at a radial distance $r$ and time $t, \rho_{\text {lid }}$ is the average lid density, $k_{\text {lid }}$ is the thermal conductivity of the lid, and $C_{\mathrm{P} \text {,lid }}$ is the specific heat capacity of the lid. The boundary conditions applied are a prescribed fixed temperature at the outer surface of the lid $\left(T_{\text {lid }}\left(R_{\text {surf }}, t\right)=T_{\text {surf }}\right)$; and a prescribed time-dependent heat flux at the interface between the 
lid and underlying mantle $\left(-k_{\text {lid }} \frac{\partial T_{\text {lid }}}{\partial r}=q_{\text {man-top }}(t)\right)$. The conductive lid is thermally coupled to the underlying convective mantle through the thermal boundary conditions, where the bottom heat flow is obtained from the convection model for the mantle. The time-dependent bottom temperature of the lid, on the other hand, is applied as a boundary condition for the convecting mantle part of the domain.

Equation (10) is solved numerically by a finite-difference discretization method using 100 grid points in the radial direction (van Kan et al. 2014). Time discretization then results in a system of algebraic equations that are solved with a time stepping algorithm that combines the solution of the conductive lid and the convecting mantle coupled through the boundary conditions.

\subsection{Modeling assumptions}

In this section, modeling assumptions for the mantle evolution and coupled core-mantle-lid setups are provided. Tables 1 and 2 list the material and planet properties used for modeling.

\subsubsection{Material properties}

All relevant material properties concerning Eqs. (3)-(10) for graphite, silicate, and iron are given in Table 1. For the viscosity of graphite, the strain rate equation from Wagner \& Driesner (1959) is implemented, which gives the rheology prefactor $A$ in terms of the shear modulus $\mu$ and corresponding prefactor $B=1.75$ as $A=\mu / 2 B$. The shear modulus of graphite has been reported to be as low as $10 \mathrm{GPa}$ (Cost et al. 1968) and as high as $350 \mathrm{GPa}$ (Min \& Aluru 2011). For this reason we implement two end-member rheology prefactors for graphite (Table 1). For thermal conductivity of graphite, we used the Hofmeister et al. (2014) model with a temperature dependence given in Table 1. However, we also quantified the effect of a temperatureindependent thermal conductivity of graphite in Sect. 3.1.

\subsubsection{Mantle evolution setup properties}

In Table 2, we list all models implemented in Sect. 3. The mantle evolution setup (Fig. 1a) is used in Sect. 3.1 to illustrate that a graphite mantle exits the convection regime of heat transport and enters the conductive regime much earlier than a silicate mantle. For this purpose, we defined the duration of convective cooling as the time required for the Nusselt number to reach unity. We integrated Eq. (7) to compute the mantle temperature evolution supplemented by Eqs. (3)-(6) and parameter values for graphite or silicate from Table 1. We kept the core size of our model fixed at $1500 \mathrm{~km}$ and the mantle thickness the same for the graphite and silicate cases (see Table 2). This setup allowed us to isolate the effects of planet properties such as the planet size, surface area, and gravity or internal heating and initial temperature on our model outcomes. For the base case, we assumed a surface temperature of $700 \mathrm{~K}$ (see Table 1), identical initial mantle temperatures of $2000 \mathrm{~K}$, and no radiogenic heating. To quantify the effects of initial mantle temperature, radiogenic heating, and thermal conductivity model of graphite, we varied these parameters one by one (see Sect. 3.1).

\subsubsection{Coupled core-mantle-lid evolution setup properties}

In Sects. 3.2, 3.3, and 3.4, we implemented the coupled coremantle-lid evolution setup (Fig. 1b). We assumed the core to be made of iron, the mantle to contain silicates, and the lid (if present) to be either silicate or graphite. We also implemented reference cases with extremely thin lid to simulate lidless planets in Sects. 3.3 and 3.4. We integrated Eqs. (8)-(10) for the three layers (Fig. 1b) to calculate the thermal evolution. To isolate model-dependent effects, we fixed the core and mantle radii at 1500 and $3000 \mathrm{~km}$, respectively, and only varied the lid thickness (Table 2). Across different models, we also assumed the same surface temperature, the same initial temperatures for the core, mantle, and lid, and the same heating rate for the mantle (see Model properties in Table 1). We assumed the internal heating in the core and lid to be zero. In Sect. 3.4, we implemented this setup to Kepler-37b with a known radius of $2166 \mathrm{~km}$ (Stassun et al. 2017). We fixed the core radius of our Kepler-37b models to half of the total radius and mantle and the lid thicknesses varied depending on the model (Table 2).

\section{Results}

\subsection{Duration of convective cooling in graphite and silicate mantles}

Our calculations implementing the mantle evolution setup (no lid) in Fig. 1a show that the duration of convective cooling (see Sect. 2.3.2) for silicate-mantle planets with mantle thicknesses between $100-1000 \mathrm{~km}$ is between $0.05-3.7 \mathrm{Gyr}$ (Fig. 2). In contrast, the convective cooling duration for graphite-mantle planets is an order of magnitude lower $(0.006-0.34$ Gyr). For graphitemantle planets, the cases of minimum and maximum shear modulus (see Sect. 2.3.2) differ by $0.3 \mathrm{Myr}$ (for the $100 \mathrm{~km}$ case) and 4.6 Myr (for the $1000 \mathrm{~km}$ case), implying a negligible effect of shear modulus on the cooling of the planet. If we adopt a constant thermal conductivity of graphite $\left(40 \mathrm{~W} \mathrm{~m}^{-1} \mathrm{~K}^{-1}\right)$ instead of the Hofmeister et al. (2014) model, the duration of convective cooling decreases by $20-35 \%$. This is because the Hofmeister thermal conductivity is lower than $40 \mathrm{~W} \mathrm{~m}^{-1} \mathrm{~K}^{-1}$ at initial graphite mantle temperature considered in this work (see inset Fig. 2).

Assuming an initial mantle temperature of $4000 \mathrm{~K}$ instead of $2000 \mathrm{~K}$ increases the lifetime of convection for the silicate and graphite cases by only 10-80 Myr and 0.7-5 Myr, respectively. Incorporating internal heating (see Table 1), we find that silicate-mantle models need only up to $0.2 \%$ more time to reach $\mathrm{Nu}=1$ compared to the models without internal heating. As the main radiogenic heat producing elements in rocky planets $(\mathrm{U}, \mathrm{Th}$, and $\mathrm{K})$ are highly incompatible in graphite, it is unlikely that significant internal heating in a graphite layer would occur under any circumstances. Even if radiogenic heating in the graphite mantle is made equal to that in silicates, the duration of convective cooling changes by less than $0.1 \%$.

Although we ignore the pressure-dependent term in viscosity in our modeling, we extend our calculations to larger planets up to the size of Earth. Our calculations show that for large planets the duration of convective cooling increases by less than $20 \%$ compared to the planets shown in Fig. 2 for layer thicknesses up to a few hundred kilometers. Fast cooling of a graphite layer is attributed to the high thermal conductivity of graphite.

\subsection{Thermal evolution of planets with graphite and silicate lids}

Because of its high thermal conductivity and efficient cooling, a physically separate graphite outer shell inevitably acts as an insulating stagnant lid on top of a silicate mantle. In contrast, a silicate lid may form on top of the convecting mantle as a 


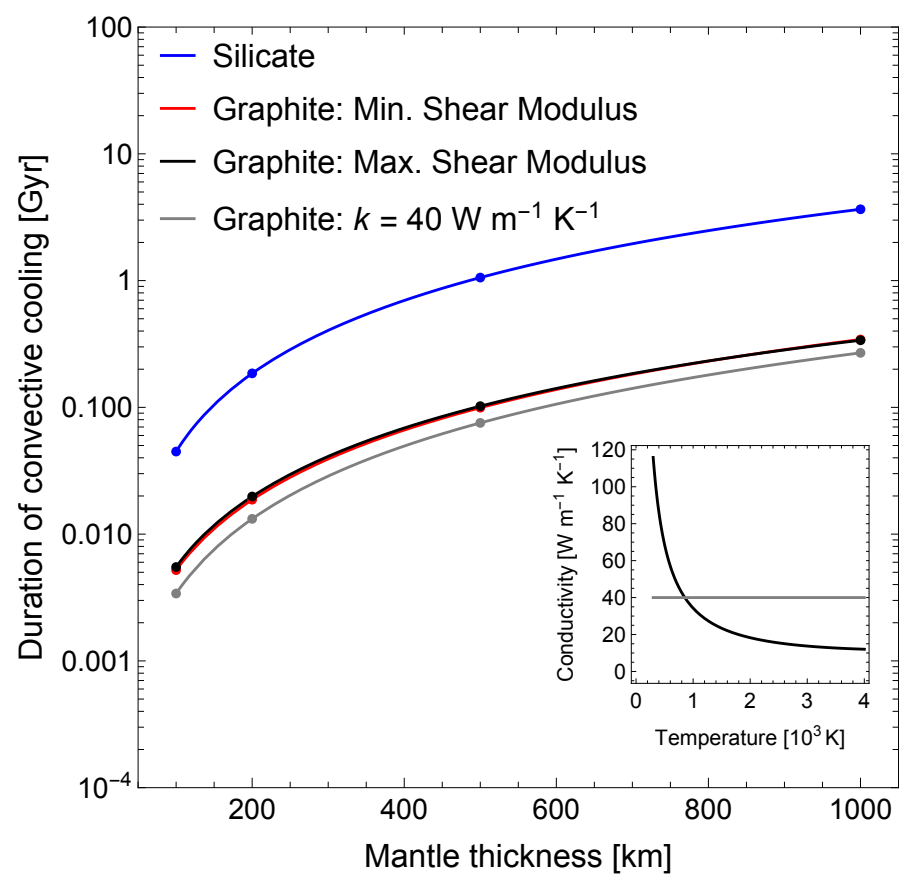

Fig. 2. Comparison of the convective cooling duration of graphite and silicate mantles with a core radius of $1500 \mathrm{~km}$ and a mantle thickness of $100-1000 \mathrm{~km}$. The inset panel shows the two models (Hofmeister et al. 2014, and constant thermal conductivity) for thermal conductivity of graphite used to calculate the duration of convective cooling. For models with a graphite mantle, in addition to constant thermal conductivity, two cases of viscosity based on the minimum and maximum values of shear modulus are also compared.

consequence of the temperature-dependence of viscosity. The thickness of the silicate lid depends on the thermal state of the planet and increases as the planet cools. For a hot mantle and/or a large carbon inventory, the graphite outer shell could be much thicker than what the silicate lid would be. In particular planets with plate tectonics, such as Earth, do not exhibit a stagnant lid. To evaluate the effect of an outer graphite shell on the cooling rate, in this section we first compare planets with a fixed lid thickness made of either graphite or silicate. In a second step, we compare planets with different graphite lid thicknesses with each other (Sect. 3.3). Finally, we compare the thermal evolution of Kepler-37b assuming a graphite lid or a silicate lid or no lid (Sect. 3.4).

Implementing the coupled core-mantle-lid setup (Fig. 1b), we compare the thermal evolution of planets with either a graphite lid or a silicate lid and a lid thickness of $50 \mathrm{~km}$. See Tables 1 and 2 and Sect. 2.3.2 for material properties and modeling assumptions. The iron core and silicate mantle radii are fixed at 1500 and $3000 \mathrm{~km}$. The internal heating rate is the same for both models. It is well known that the presence of a stagnant lid on top of a convective mantle delays the cooling of the mantle. We are interested in the differences in planetary cooling due to different lid compositions.

Figure 3 compares several properties related to planetary thermal evolution spanning 5 Gyr. Despite the same initial temperatures for both models, there is a significant difference in the evolution of temperature (Fig. 3a). For the two cases the temperature at the bottom of the lid differs by almost $400 \mathrm{~K}$ and the core and mantle temperatures differ by more than $100 \mathrm{~K}$. These differences between the two models are attributed to up to an order of magnitude difference in the thermal conductivity of graphite and silicate lids (Fig. 3f). The initial thermal conductivity distribution within the graphite lid varies between $20-50 \mathrm{~W} \mathrm{~m}^{-1} \mathrm{~K}^{-1}$ because of the large distribution (a difference of $1000 \mathrm{~K}$ between the top and bottom of the lid) in the initially assumed lid temperature profile (Fig. 3e). Although the initial temperature distribution within the lid is the same for silicate and graphite lids, the higher thermal conductivity of graphite cools the graphite lid faster than the silicate lid. A drop in the temperature of graphite increases its thermal conductivity and makes its thermal conductivity distribution in the lid flatter (see $200 \mathrm{Myr}$ and $5 \mathrm{Gyr}$ profiles in Fig. 3f). This is a direct consequence of the inverse temperature proportionality of thermal conductivity of graphite in the Hofmeister model (inset Fig. 2). This increased thermal conductivity of graphite lid further accelerates cooling of the lid.

The lower the temperature at the bottom of the lid, the higher is the temperature contrast across the thermal boundary layer at the top of the mantle. In the graphite lid case, this higher temperature contrast allows for a higher heat flux through the top of the mantle especially in the first $600 \mathrm{Myr}$ (see Fig. 3b). Consequently, higher heat flux allows the mantle and the core to cool faster. We note that between $0.2-1.8 \mathrm{Gyr}$ the heat flux in the silicate lid case at the bottom of the mantle is lower than that in the graphite lid case (Fig. 3b) because of a lower temperature contrast between the core and mantle temperature at the bottom thermal boundary layer (Fig. 3a). As mantle viscosity is a function of the mantle temperature, it increases rapidly with time for the graphite lid case compared to the silicate lid case as seen in Fig. 3c. The minimum in the mantle viscosity in the silicate lid case at about $0.3 \mathrm{Gyr}$ arises from the corresponding maximum in the mantle temperature in Fig. 3a. The Nusselt number also decreases faster for the graphite lid case than for the silicate lid case (Fig. 3d).

Clearly, a $50 \mathrm{~km}$ silicate lid significantly delays the cooling of a planet compared to a $50 \mathrm{~km}$ graphite lid. Another relevant comparison between silicate and graphite lids is to quantify the equivalent thickness of a silicate lid to achieve the same cooling as a graphite lid. In Fig. 4a, each data point represents two models: one with a graphite lid and another with a silicate lid, which have the same temperature at the bottom of the lid after $5 \mathrm{Gyr}$ of evolution. We plot this lid-bottom temperature in Fig. 4b. A silicate lid with approximately an order of magnitude lower thickness than the graphite lid is sufficient to reproduce the same temperature at the bottom of the lid after 5 Gyr. This is a significant result because it implies that a planet with a graphite lid cools similar to a planet with a silicate lid that is approximately ten times thinner.

\subsection{Effects of graphite lid thickness on thermal evolution}

In Sect. 3.2, we show that silicate lids are significantly more inefficient at planetary cooling than graphite lids. In this section, we model the thermal evolution of planets that do not form a silicate lid. We quantify the effect of graphite lid thickness on thermal evolution by implementing the coupled core-mantle-lid setup. We fix the core and mantle radii at 1500 and $3000 \mathrm{~km}$, and add graphite lids with thicknesses of 1,50 , and $500 \mathrm{~km}$ on top of the mantle. The $1 \mathrm{~km}$ case is introduced to emulate a planet without a conductive lid and to remove any model dependences such as the temperature contrast at the top of the mantle. Again the total internal heating is the same as it depends on the volume of the mantle, which is the same for all models.

Figure 5a shows that the silicate mantle and graphite lid of the $50 \mathrm{~km}$ model cool slower than the $1 \mathrm{~km}$ model because of several effects. First, the thermal inertia of the graphite lid, 

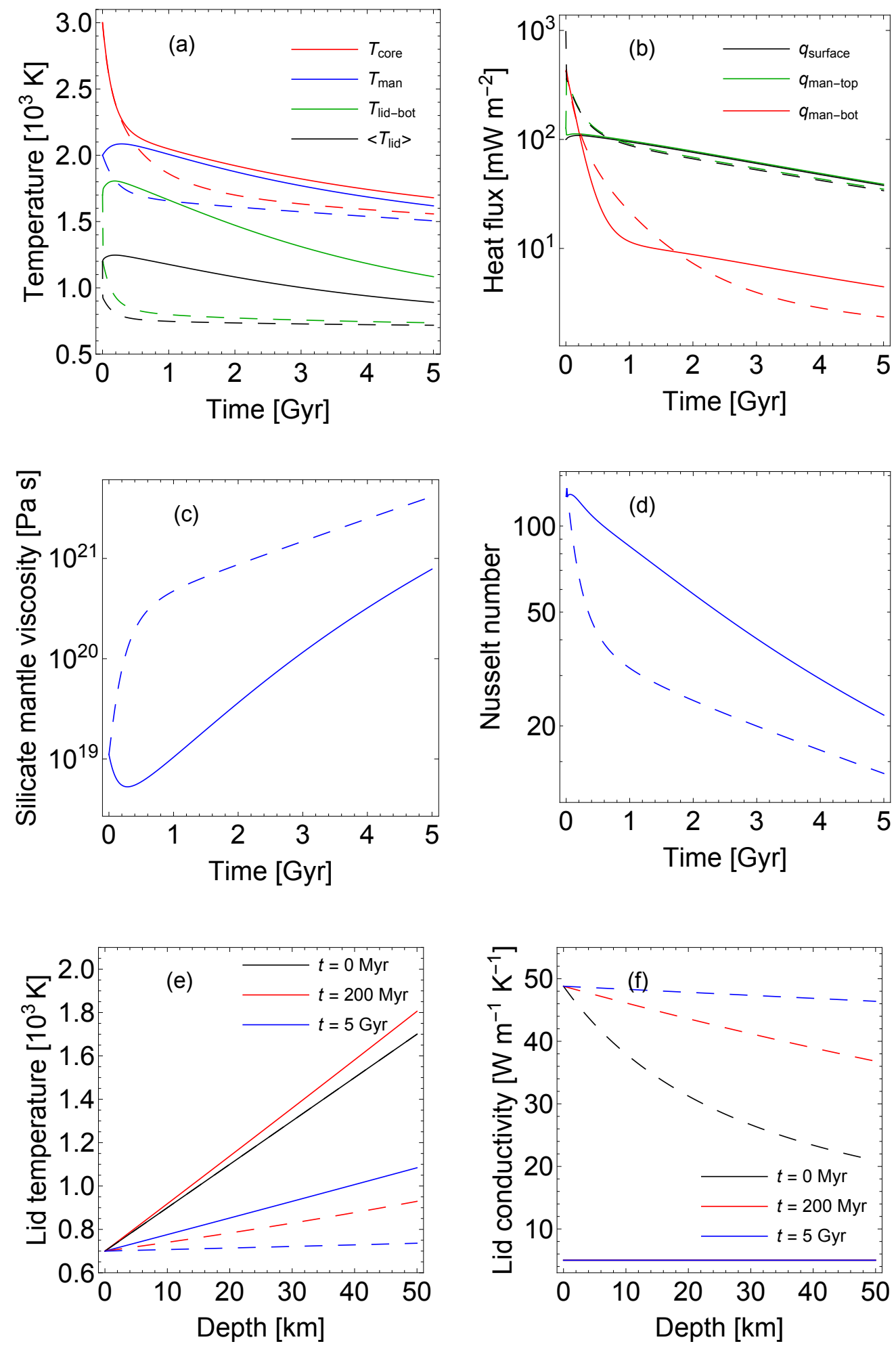

\section{0 km Silicate Lid - - 50 km Graphite Lid}

Fig. 3. Coupled core-mantle-lid thermal evolution for models with either a graphite or silicate lid. The core, mantle, and planetary radii are identical for the two models. The $0 \mathrm{Myr}$ lines overlap in plot $e$.

which is related to its heat capacity and its thermal conductivity, smoothens a rapid temperature drop in the early stages. Second, the graphite lid presents a thermal resistance that reduces the surface heat flux for a given temperature contrast (see van den Berg et al. 2005). Third, the presence of an outer shell reduces the temperature contrast at the top of the mantle, which drives thermal convection. Compared to the $50 \mathrm{~km} \mathrm{C}$-lid, the $500 \mathrm{~km}$ $\mathrm{C}$-lid provides both a much larger thermal inertia and thermal resistance (hereafter, collectively termed as thermal shielding) resulting in much longer cooling times for its silicate mantle. Although the lid-bottom temperature and the silicate mantle temperature of the $500 \mathrm{~km}$ model also tend to approach the 

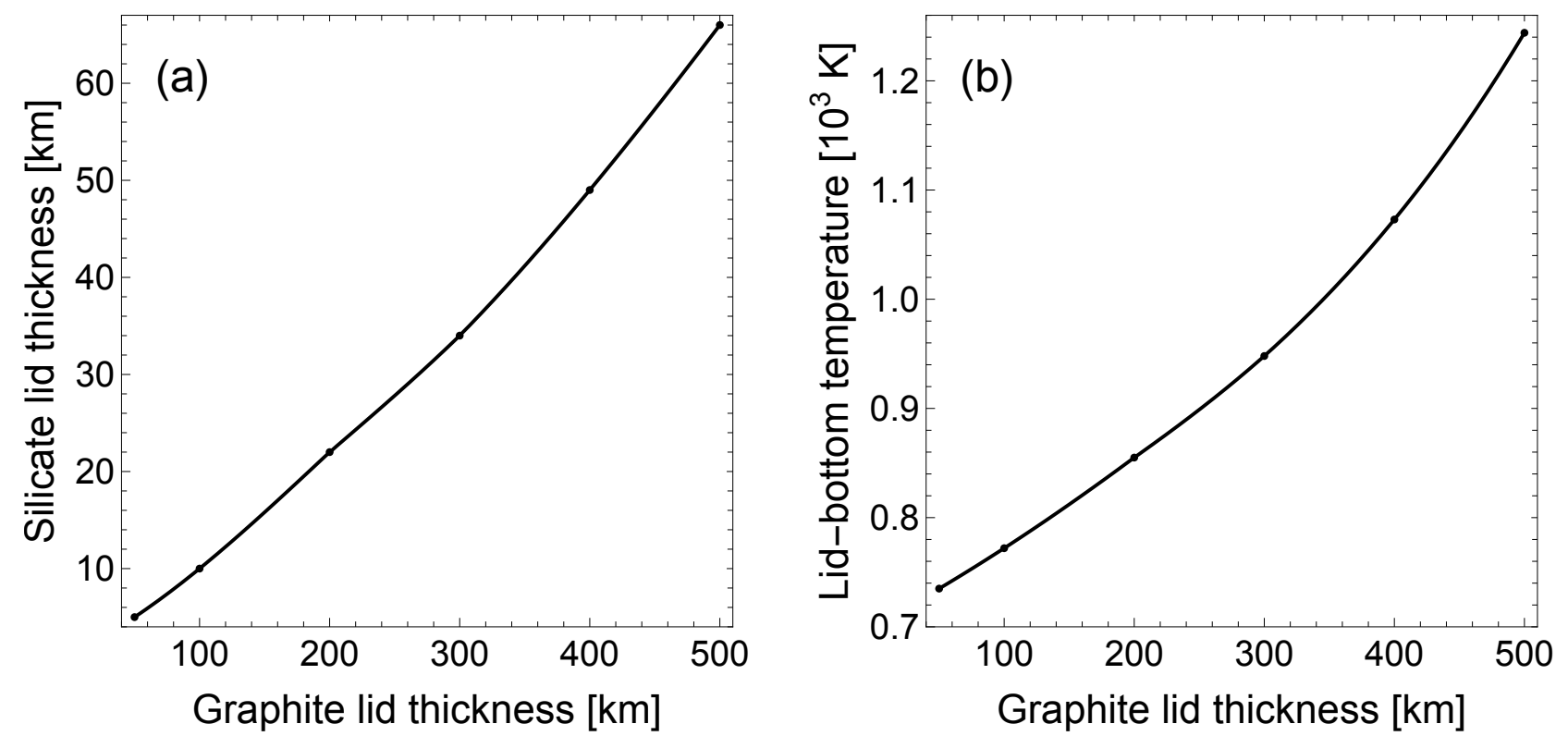

Fig. 4. (a) Thicknesses of silicate and graphite lids required to reach the same temperature at the bottom of the lid after 5 Gyr of evolution. (b) Corresponding temperature at the bottom of the lid after $5 \mathrm{Gyr}$ of evolution. The initial lid-bottom temperature is $1700 \mathrm{~K}$ in all cases.

respective temperatures of the reference model after 5 Gyr, they are still higher than the other two models by about 500 and $200 \mathrm{~K}$ at $5 \mathrm{Gyr}$, respectively (Fig. 5a). Our calculations for $100-500 \mathrm{~km} \mathrm{C}$-lid cases indicate that a thin graphite shell $(<200 \mathrm{~km})$ exhibits small thermal shielding and does not significantly affect the long-term thermal evolution.

In Fig. 5b, the heat flux at the bottom of the mantle for the $50 \mathrm{~km}$ case is smaller than that of the $1 \mathrm{~km}$ case between 0.5-4 Gyr. It is consistent with the smaller core-mantle temperature contrast for the $50 \mathrm{~km}$ case shown in Fig. 5a. The smaller drop of the core-mantle temperature contrast corresponds to a steeper drop in the core temperature in the first 1 Gyr combined with a smoother drop in the temperature of the lid, which is related to the latter. In contrast, the $1 \mathrm{~km}$ case shows a higher core-mantle temperature contrast in line with the absence of the thermal inertia of the $\mathrm{C}$-lid. For the $500 \mathrm{~km}$ lid model, during the first $0.4 \mathrm{Gyr}$ the drop in the core temperature is similar to that of the $50 \mathrm{~km}$ case. However, after $0.4 \mathrm{Gyr}$, the core temperature decreases slowly as a consequence of the thermal shielding effect of the thick graphite lid, not allowing the heat to escape from the core efficiently. This results in a higher core temperature (by $200 \mathrm{~K}$ ) for the $500 \mathrm{~km}$ lid model at $5 \mathrm{Gyr}$ than in the other two models. The minimum in the core heat flux for the $500 \mathrm{~km}$ case at $0.9 \mathrm{Gyr}$ corresponds to a maximum of the mantle temperature and almost disappearing temperature contrast at the core-mantle boundary. We could speculate that such an event might lead to the demise of an early planetary magnetic field through a shutdown of a core-dynamo process (e.g., Stevenson 2001).

The trends in the silicate mantle temperature corroborate the trends in the silicate mantle viscosity and the Nusselt number as seen in Figs. 5c,d. Figure 5e shows three snapshots of the radial temperature profile in the graphite lid. For the $50 \mathrm{~km}$ C-lid model, the temperature profile at $5 \mathrm{Gyr}$ is not as steep as at $200 \mathrm{Myr}$ as expected from the evolution of temperature at the bottom of the lid. For the $500 \mathrm{~km}$ model, since the lid temperature increases first and then decreases, its $200 \mathrm{Myr}$ temperature profile crosses the initial profile. This effect is again a result of the large thermal shielding provided by the $500 \mathrm{~km}$ C-lid. Similar trends are observed for the radial distribution of thermal conductivity in the graphite shell (Fig. 5f).

\subsection{Application to Kepler-37b}

We now apply the coupled core-mantle-lid setup (Fig. 1b) to Kepler-37b to demonstrate differences in the thermal evolution of lidless, graphite-lid, and silicate-lid cases. For this purpose, we use three models, $1 \mathrm{~km}$ graphite-lid (emulating a planet without any lid), $100 \mathrm{~km}$ graphite-lid, and $100 \mathrm{~km}$ silicate-lid, respectively (Table 2). The core radius is fixed to half of the total radius. Although the thickness of a stagnant lid evolves with time, in this case we use models with a fixed stagnant lid thickness. This is required for a model-independent comparison between graphite and silicate lids.

Qualitatively, the three cases shown in Fig. 6 are similar to the three cases described in Sect. 3.3. As expected, a lidless planet cools the fastest, followed by the $100 \mathrm{~km}$ graphite-lid model, whereas the silicate-lid Kepler-37b model significantly slows down cooling, essentially behaving like a much thicker graphite-lid model as demonstrated in Fig. 5. The core temperature of the $100 \mathrm{~km}$ C-lid case in Fig. 6a is close to that of the $1 \mathrm{~km}$ $\mathrm{C}$-lid case. For the $100 \mathrm{~km}$ silicate lid case, the core temperature stays above the temperatures of the other two cases because of intense thermal shielding. The core heat flux in Fig. 6b is smallest for the $100 \mathrm{~km}$ silicate lid case in the first $1.8 \mathrm{Gyr}$ in line with the small core-mantle temperature contrast and an initially increasing mantle temperature. Since all radiogenic heating in the model is assumed to be concentrated in the mantle silicates and specified at the same initial value per unit mass (Table 1), the amount of internal heating decreases with the increasing thickness of the lid. That is why the total internal heating in the $100 \mathrm{~km}$ silicate and graphite lid models is the same but is about $14 \%$ lower initially than the $1 \mathrm{~km} \mathrm{C}$-lid model Fig. 6d. However, this difference in internal heating has a small effect that is not discernible between the 1 and $100 \mathrm{~km}$ C-lid models.

Silicate mantle viscosities are lower for models with higher thermal shielding (Fig. 6c). Because of the direct dependence of viscosity on the mantle temperature, a local minimum is observed in the $100 \mathrm{~km}$ silicate-lid case owing to a corresponding temperature maximum. The Rayleigh and Nusselt numbers of the $100 \mathrm{~km} \mathrm{C}$-lid model end up higher than those of the $1 \mathrm{~km} \mathrm{C}$-lid model because of their strong dependence on the 
K. Hakim et al.: Thermal evolution of rocky exoplanets with a graphite outer shell
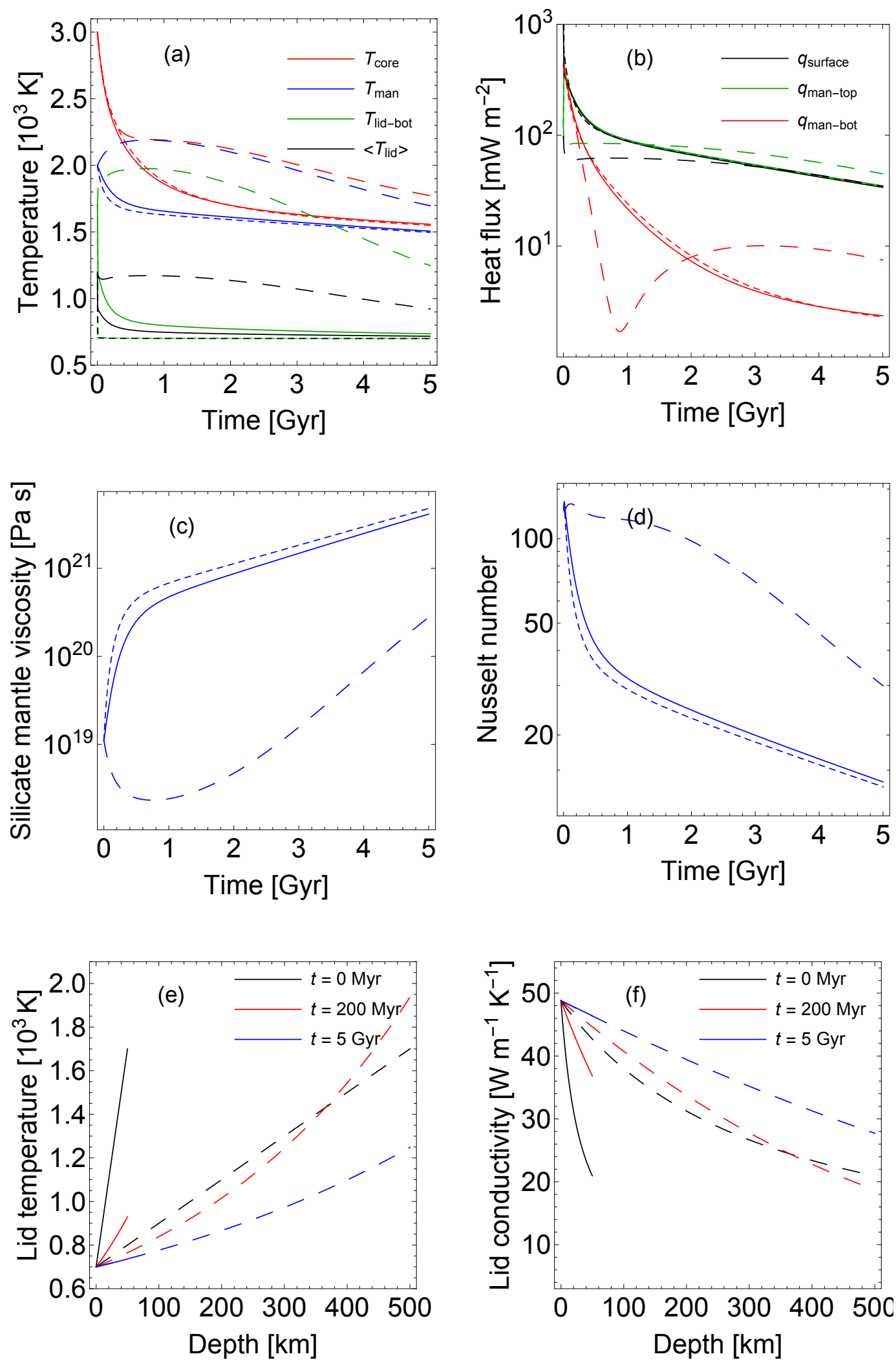

$---1 \mathrm{~km} \mathrm{C-lid}-50 \mathrm{~km} \mathrm{C-lid} \mathrm{-} 500 \mathrm{~km} \mathrm{C-lid}$

Fig. 5. Parameters related to the thermal evolution of three-layer planets with core and mantle radii of 1500 and $3000 \mathrm{~km}$, respectively. All three models have different graphite lid thicknesses $(1,50$, and $500 \mathrm{~km})$ and consequently different planetary radii. In plots $e$ and $f$, the $5 \mathrm{Gyr}$ lines for the 50 and $500 \mathrm{~km}$ cases overlap; the $1 \mathrm{~km}$ case is not shown. 

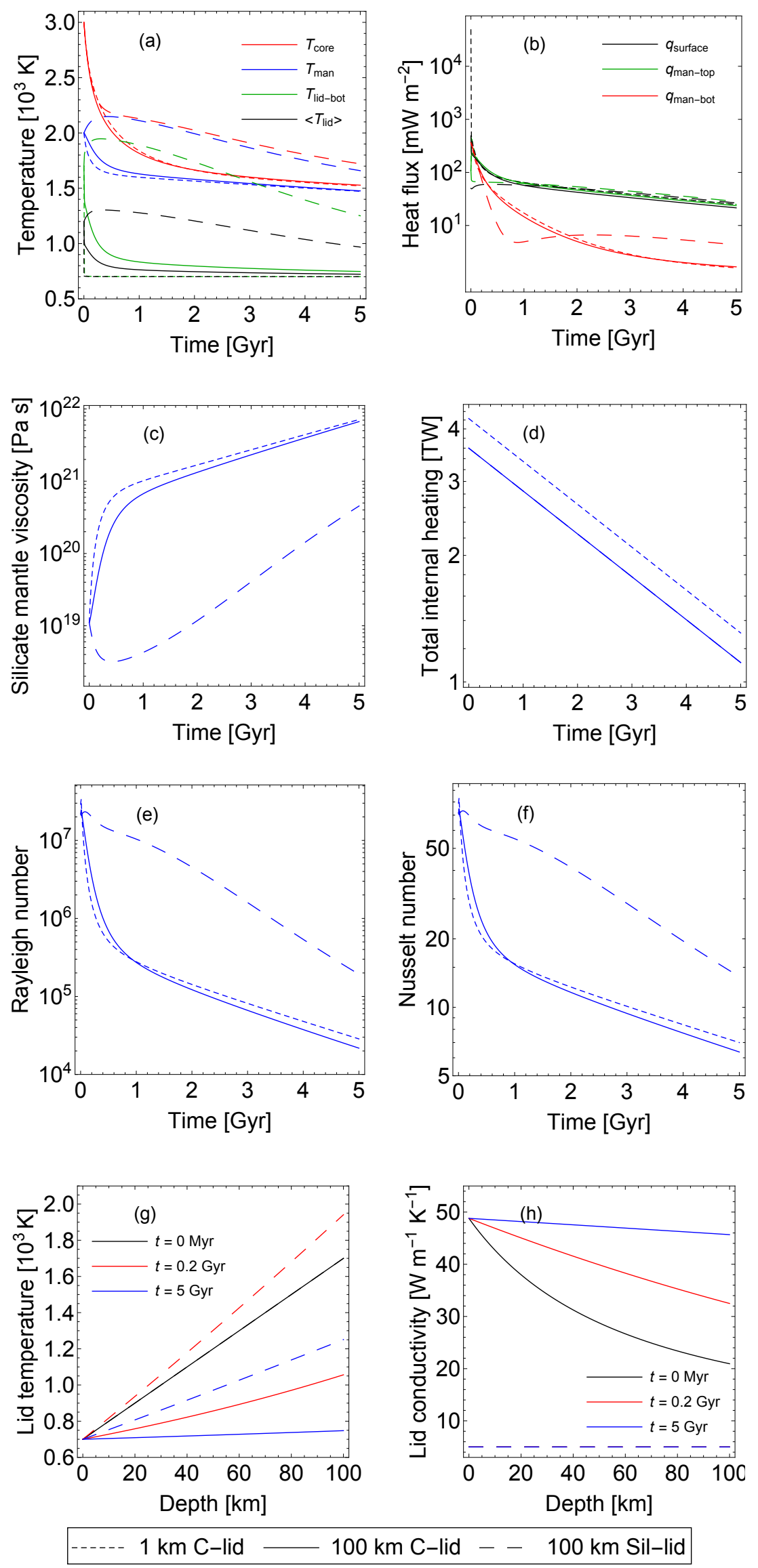

Fig. 6. Thermal evolution parameters of Kepler-37b for models with $100 \mathrm{~km}$ thick silicate and graphite lids compared to a $1 \mathrm{~km}$ graphite-lid model. In plot $g$ the $0 \mathrm{Myr}$ lines overlap; the $1 \mathrm{~km}$ case is not shown in plots $g$ and $h$. 
mantle thickness, which is smaller for the $100 \mathrm{~km} \mathrm{C}$-lid model (Figs. 6e,f). On the other hand, the Rayleigh and Nusselt numbers of the $100 \mathrm{~km}$ silicate-lid models are higher than those of the other two models because of the difference in their viscosity. The trends in the radial distribution of temperature and thermal conductivity (Figs. 6g,h) in the lid are similar to those in Sect. 3.3.

\section{Discussion and conclusions}

In this paper, we model the thermal evolution of rocky exoplanets whose chemical composition and physical structure are different from those of the terrestrial planets we know of. Not surprisingly, the thermal structure depends on the mineralogy of different layers in the planet. Carbon-enriched rocky exoplanets are expected to contain an iron core, a silicate mantle, and a graphite outer shell (Hakim et al. 2019). Our calculations show that a graphite layer is largely conductive in nature during all but the earliest stages of planetary evolution, essentially behaving like a stagnant lid with a fixed thickness. This is mainly a result of thermal conductivity of graphite being approximately one order of magnitude higher than that of common mantle silicate minerals. For the same reason, a conductive silicate lid would slow down cooling by as much as an order of magnitude thicker conductive graphite lid would do. As such our models are applicable to stagnant lid planets with different lid thermal conductivities. For example, if Mercury has a stagnant lid partially consisting of graphite in addition to silicates, its cooling might have been accelerated compared to the assumption of fully silicate lid. On the other hand, for a Mars-size planet, a $100 \mathrm{~km}$ lid model with half the thermal conductivity of graphite would end up with a $100 \mathrm{~K}$ higher temperature at the bottom of the lid at 5 Gyr than the $100 \mathrm{~km}$ graphite-lid model. Whereas, a $100 \mathrm{~km}$ model with a diamond-like thermal conductivity would cool as fast as the $1 \mathrm{~km}$ graphite-lid model.

As opposed to a planet without any stagnant lid, a graphite lid slows down the cooling of the planet by thermally shielding the interior due to the thermal inertia and thermal resistance of the graphite lid. The thermal inertia is mostly important during the first $\sim 100 \mathrm{Myr}$ of planetary evolution when the thermal profile of the lid changes fast. The thermal resistance of the graphite lid (e.g., van den Berg et al. 2005) controls the longterm thermal evolution. We find that a thin outer graphite shell $(<200 \mathrm{~km})$ has a small effect on the heat release from the deep interior of the planet. This implies that a thin graphite lid on top of the silicate mantle does not significantly impact the longterm evolution of the interior. However, for planets with higher graphite layer thicknesses, the thermal shielding effect of the lid becomes significant enough to slow down the cooling of the planet by several billion years. With the application to Kepler$37 \mathrm{~b}$, we show that a lidless model cools faster than a graphite-lid model, which cools faster than a silicate-lid model.

Our models do not take into account the temperature and pressure effects on the heat capacity of graphite or the pressure effects on thermal conductivity and viscosity. To assess hightemperature effects, our chosen parameter values are either based on ambient temperature data or measurements in a temperature range relevant to our models depending on the availability of temperature-dependent values of the parameter. Since the interior pressures of planets considered in this study are relatively small, we expect these effects to be small and to not change our conclusions in a qualitative way for planets at least up to the size of Mars. For example, the pressure-dependent silicate viscosity for Kepler-37b-size planets is only $10 \%$ higher than the pressure-independent silicate viscosity. On the other hand, to our knowledge, there are no experimental studies focusing on the effect of high pressure on the properties of graphite. In the future, experimental studies of the high-pressure hightemperature properties of graphite such as thermal conductivity, heat capacity, and activation parameters could further refine assessments of the effect of graphite shells on planetary thermal evolution.

Since the density of diamond is similar to mantle silicates, diamond is likely to stay mixed with silicates in the mantle, leaving the outer shell to be composed only of low-density graphite. Thus, the maximum possible thickness of the graphite outer shell for a given planet is determined by the graphitediamond transition pressure above which no graphite exists. As pressures in larger planets increase steeply with depth, the graphite-diamond transition pressure occurs at shallower depths than that for smaller planets. For example, if we assume a temperature-independent graphite-diamond transition pressure of $10 \mathrm{GPa}$, the maximum possible outer graphite shell thicknesses for a planet with a radius of $3500 \mathrm{~km}$, an Earth-size planet, and a planet twice the size of Earth would be about 1500, 400, and $100 \mathrm{~km}$, respectively.

Unterborn et al. (2014) showed that the mixing of diamond with the silicate mantle accelerates the cooling process because of the extremely high thermal conductivity of diamond. If such planets have a graphite outer shell, diamond mixing in the silicate-rich mantle would cool the planet faster while the thermal shielding effect of graphite would slow down the cooling. The net planetary cooling rate of such planets would be faster or slower compared to a lidless planet without graphite or diamond depending on the effect that dominates. For Mercury-size and smaller planets (e.g., Kepler-37b), the mantle pressures would not be high enough to stabilize diamonds.

This study exhibits thermal evolution modeling of carbonenriched rocky exoplanets that have no solar system analogs. Our calculations show that the overall cooling is greatly affected by the mineralogy of different layers in the planet. As our knowledge of atmospheric and interior composition of rocky exoplanets advances with the data from current and future telescopes (e.g., TESS, CHEOPS, JWST, ELT, PLATO, and ARIEL), the understanding of their interior and surface dynamics also needs to advance with theoretical studies such as this work.

Acknowledgements. We thank Lena Noack for a constructive review that significantly improved this manuscript. We also thank Dan Bower for his valuable comments. This work is part of the Planetary and Exoplanetary Science Network (PEPSci), funded by the Netherlands Organization for Scientific Research (NWO, Project no. 648.001.005). K.H. also acknowledges financial support from the European Research Council via Consolidator Grant ERC-2017-CoG-771 620EXOKLEIN (awarded to Kevin Heng).

\section{References}

Barclay, T., Rowe, J. F., Lissauer, J. J., et al. 2013, Nature, 494, 452 Bond, J. C., Lauretta, D. S., Tinney, C. G., et al. 2008, ApJ, 682, 1234 Bond, J. C., O’Brien, D. P., \& Lauretta, D. S. 2010, ApJ, 715, 1050 Boylan, J. 1996, Mater. World, 4, 707

Brewer, J. M., Fischer, D. A., Valenti, J. A., \& Piskunov, N. 2016, ApJS, 225, 32 Carter-Bond, J. C., O’Brien, D. P., \& Raymond, S. N. 2012, ApJ, 760, 44 Colonna, F., Fasolino, A., \& Meijer, E. J. 2011, Carbon, 49, 364 Cost, J. R., Janowski, K. R., \& Rossi, R. C. 1968, Philos. Mag., 17, 851 Delgado Mena, E., Israelian, G., González Hernández, J. I., et al. 2010, ApJ, 725, 2349

Deschamps, F., \& Lin, J.-R. 2014, Phys. Earth Planet. Inter., 229, 40

Deschamps, F., \& Sotin, C. 2001, J. Geophys. Res., 106, 5107

Dewaele, A., Datchi, F., Loubeyre, P., \& Mezouar, M. 2008, Phys. Rev. B, 77, 094106 
Dorn, C., Harrison, J. H. D., Bonsor, A., \& Hands, T. O. 2019, MNRAS, 484, 712

Fei, Y., Murphy, C., Shibazaki, Y., Shahar, A., \& Huang, H. 2016, Geophys. Res. Lett., 43, 6837

Ghiringhelli, L. M., Los, J. H., Meijer, E. J., Fasolino, A., \& Frenkel, D. 2005, Phys. Rev. Lett., 94, 145701

Hakim, K., Rivoldini, A., Van Hoolst, T., et al. 2018a, Icarus, 313, 61

Hakim, K., van Westrenen, W., \& Dominik, C. 2018b, A\&A, 618, L6

Hakim, K., Spaargaren, R., Grewal, D. S., et al. 2019, Astrobiology, 19, 867

Hofmeister, A. M. 1999, Science, 283, 1699

Hofmeister, A. M., Dong, J., \& Branlund, J. M. 2014, J. Appl. Phys., 115, 163517

Höning, D., \& Spohn, T. 2016, Phys. Earth Planet. Int., 255, 27

Höning, D., Tosi, N., \& Spohn, T. 2019, A\&A, 627, A48

Keppler, H., \& Golabek, G. 2019, Geochem. Perspect. Lett., 11, 12

Kerley, G. I., \& Chhabildas, L. C. 2001

Klarmann, L., Ormel, C. W., \& Dominik, C. 2018, A\&A, 618, L1

Kobayashi, Y. 1974, J. Phys. Earth, 22, 359

Min, K., \& Aluru, N. R. 2011, Appl. Phys. Lett., 98, 013113

Morgan, W. C. 1972, Carbon, 10, 73

Moriarty, J., Madhusudhan, N., \& Fischer, D. 2014, ApJ, 787, 81

Nabiei, F., Badro, J., Dennenwaldt, T., et al. 2018, Nat. Commun., 9, 1327

Nakajima, T., \& Sorahana, S. 2016, ApJ, 830, 159

Noack, L., Höning, D., Rivoldini, A., et al. 2016, Icarus, 277, 215

Peplowski, P. N., Klima, R. L., Lawrence, D. J., et al. 2016, Nat. Geosci., 9, 273

Petigura, E. A., \& Marcy, G. W. 2011, ApJ, 735, 41

Petigura, E. A., Marcy, G. W., Winn, J. N., et al. 2018, AJ, 155, 89

Schubert, G., Cassen, P., \& Young, R. E. 1979, Icarus, 38, 192

Schubert, G., Turcotte, D. L., \& Olson, P. 2001, Mantle Convection in the Earth and Planets (Cambridge: Cambridge University Press), 956
Seager, S., Kuchner, M., Hier-Majumder, C. A., \& Militzer, B. 2007, ApJ, 669, 1279

Spohn, T. 1991, Icarus, 90, 222

Stassun, K. G., Collins, K. A., \& Gaudi, B. S. 2017, AJ, 153, 136

Stevenson, D. J. 2001, Nature, 412, 214

Stevenson, D. J., Spohn, T., \& Schubert, G. 1983, Icarus, 54, 466

Stixrude, L., \& Lithgow-Bertelloni, C. 2011, Geophys. J. Int., 184, 1180

Turcotte, D. L., \& Oxburgh, E. R. 1967, J. Fluid Mech., 28, 29

Turcotte, D. L., \& Schubert, G. 2002, Geodynamics, 2nd edn. (Cambridge: Cambridge University Press), 472

Tyler, W. W., \& Wilson, A. C. 1953, Phys. Rev., 89, 870

Unterborn, C. T., Kabbes, J. E., Pigott, J. S., Reaman, D. M., \& Panero, W. R. 2014, ApJ, 793, 124

Valencia, D., O'Connell, R. J., \& Sasselov, D. 2006, Icarus, 181, 545

Valencia, D., O'Connell, R. J., \& Sasselov, D. D. 2007, ApJ, 670, L45

van den Berg, A. P., Rainey, E. S. G., \& Yuen, D. A. 2005, Phys. Earth Planet. Int., 149,259

van den Berg, A. P., Yuen, D. A., Beebe, G. L., \& Christiansen, M. D. 2010, Phys. Earth Planet. Int., 178, 136

van Kan, J., Segal, A., \& Vermolen, F. J. 2014, Numerical Methods in Scientific Computing (The Netherlands: Delft Academic Press/VSSD)

Wagner, P., \& Driesner, A. R. 1959, J. Appl. Phys., 30, 148

Wagner, F. W., Sohl, F., Hussmann, H., Grott, M., \& Rauer, H. 2011, Icarus, 214 366

Wei, L., Kuo, P. K., Thomas, R. L., Anthony, T. R., \& Banholzer, W. F. 1993, Phys. Rev. Lett., 70, 3764

Wolstencroft, M., Davies, J. H., \& Davies, D. R. 2009, Phys. Earth Planet Int, 176,132

Zhao, Y., de Vries, J., van den Berg, A. P., Jacobs, M. H. G., \& van Westrenen W. 2019, Earth Planet. Sci. Lett., 511, 1 